\title{
MiR-499-5p Contributes to Hepatic Insulin Resistance by Suppressing PTEN
}

\author{
Lei Wang ${ }^{a}$ Ning Zhang ${ }^{b}$ Hua-ping Panc Zun Wanga Zhen-yu Cao ${ }^{a}$ \\ aDepartment of Rehabilitation Medicine, Second Medical School of Nanjing University of Chinese \\ Medicine, Nanjing, ${ }^{b}$ Department of Cardiology, Nanjing Drum Tower Hospital, Nanjing University \\ Medical school, Nanjing, 'Department of Rehabilitation Medicine, The Affiliated Jiangning Hospital of \\ Nanjing Medical University, Nanjing, China
}

\section{Key Words}

MiR-499-5p • Insulin resistance • PTEN • Type 2 diabetes

\begin{abstract}
Background: Type 2 diabetes afflicts 95\% of diabetes patients. Recent data suggest that miRNAs play a key role in insulin production, secretion and function. This study aims to explore the specific role of miR-499-5p in hepatic insulin resistance. Methods: The miRNA expression levels in the livers of $\mathrm{db} / \mathrm{db}$ mice were analyzed using miRNA chips and were verified by realtime PCR. miR-499-5p mimics and an inhibitor were transfected into NCTC1469 cells. Then, the PI3K/AKT signaling pathway and glycogen level were determined. The target genes of miR499-5 $p$ were predicted by bioinformatics and then confirmed by dual luciferase reporter assay and Western blot. To establish an insulin resistance (IR) animal model, C57BL/6 mice were fed a high-fat diet (HFD). The level of miR-499-5p in the livers of HFD-fed mice was upregulated through tail vein injection of adenovirus vectors (ad) containing miR-499-5p mimics. The glucose tolerance test (GTT) and insulin tolerance test (ITT) were used to determine glucose tolerance and insulin tolerance, respectively. Results: MicroRNA chips and qPCR showed that miR-499-5p was significantly decreased in the livers of $\mathrm{db} / \mathrm{db}$ mice. Downregulation of miR499-5p impaired the insulin signaling pathway and glycogen synthesis, whereas upregulation of miR-499-5p promoted the insulin signaling pathway and glycogen synthesis in NCTC1469 cells. The dual luciferase reporter assay and Western blot demonstrated that PTEN was the target gene of miR-499-5p. Compared with the control group, miR-499-5p was increased 2.1fold in the livers of HFD-fed mice. By tail vein injection of adenovirus vector containing miR499-5p mimics, GTT and ITT were improved in HFD-fed mice. Conclusion: Downregulation of the miR-499-5 $p$ level impaired the PI3K/AKT/GSK signaling pathway and glycogen synthesis by targeting PTEN.
\end{abstract}




\section{Cellular Physiology Cell Physiol Biochem 2015;36:2357-2365 \begin{tabular}{l|l} 
and Biochemistry Published online: July 27, 2015 & $\begin{array}{l}\text { C 2015 S. Karger AG, Basel } \\
\text { www.karger.com/cpb }\end{array}$ \\
\hline
\end{tabular} \\ Wang/Zhang/Pan/Wang/Cao: MiR-499-5p Participates in Insulin Resistance}

\section{Introduction}

The prevalence of diabetes mellitus is increasing due to environmental and genetic factors. In industrialized countries, diabetes is the leading cause of blindness, renal failure and cardiovascular disease [1]. Diabetes mellitus is characterized by increased blood glucose levels. Type 1 diabetes (T1D) is mainly caused by a loss of function of pancreatic $\beta$-cells, whereas type 2 diabetes (T2D) is caused by absolute or relatively insufficient insulin. Over $90 \%$ of all diagnosed cases are T2D, accompanied by elevated fasting blood glucose and impaired glucose tolerance [2].The liver is considered as an important insulin sensitive organ, which plays key roles in glucose homeostasis. Hepatic insulin resistance will finally lead to abnormal metabolic gene expression and impaired glyco-metabolism [3].

As a well-known tumor suppressor, phosphatase and tensin homolog deleted on chromosome 10 (PTEN) can dephosphorylate numerous proteins and phosphoinositides [4]. Studies have indicated that deletion of PTEN in livers results in enhanced glucose metabolism in mice [5]. Peripheral insulin can directly activate phosphoinositide 3-kinase (PI3K) signaling, which then regulates glucose homeostasis through the production of phosphatidylinositol 3,4,5-trisphosphate (PIP3). In this process, PTEN negatively regulates PI3K signaling by dephosphorylating PIP3 [6].

MicroRNAs (miRNA, miR) are endogenous non-coding single-stranded RNAs with approximately 22 nucleotides. Recently, miRNAs, such as miR-15, miR-122 and miR-379, have been considered as key elements in glucose metabolism [7-9]. Abnormal hepatic miRNA expression has also been identified in non-alcoholic fatty liver disease, such as miR200a, miR-200b and miR-200c [10]. In gastric cancer, colorectal carcinoma and lung cancer, miR-499-5p was found to be down-regulated and acted as a tumor suppressor by targeting forkhead box P4 (FOXP4) [11]. Recently, miR-499-5p was reported to be decreased in pancreatic islets from rodent models with insulin resistance. Through targeting apoptosisassociated tyrosine kinase (AATK), miR-499-5p could compensate $\beta$-cell mass expansion [12]. However, the functional role of miR-499-5p in hepatic insulin resistance remains unclear. In the present study, we investigated the molecular mechanism by which miR-499$5 p$ regulated insulin resistance.

\section{Materials and Methods}

\section{Animal treatment}

The $\mathrm{db} / \mathrm{db}$ mice were obtained from Peking University Health Science Center. $\mathrm{db} / \mathrm{db}$ mice $(\mathrm{n}=5)$ and age-matched wild-type (WT) mice $(n=5)$ were fed a standard laboratory diet for 4 weeks.

Four-week-old male C57BL/6J mice were also purchased from the Peking University Health Science Center. C57BL/6J mice were fed a standard laboratory diet or high-fat diet for 10 weeks in a temperature and humidity-controlled environment. A 12 h/12 h light/dark cycle was maintained. The C57BL/6J mice fed a high-fat diet (HFD-fed mice) were injected intravenously through the tail vein with adenovirus expressing miR-499-5p mimics or with a negative control adenovirus vector. The mice were anesthetized on day 7 after adenovirus injection, and blood was collected. The mouse protocols were approved by the Animal Ethics Committee at the Second Medical School of Nanjing University of Chinese Medicine.

Adenovirus vector construction

Recombinant adenoviruses expressing miR-499-5p mimic (Ad-miR-499-5p-m) or negative control adenovirus vector containing GFP (Ad-Con) were purchased from Shanghai Ribo Biotech Co., Ltd.

Metabolic phenotyping (body weight, GTT, ITT)

Body weight was measured twice weekly throughout the study. An intraperitoneal insulin tolerance test (ITT) using a dose of $0.5 \mathrm{U} / \mathrm{kg}$ insulin (Insuman ${ }^{\circledR}$ Rapid, Sanofi Aventis, Berlin, Germany) and an intraperitoneal glucose tolerance test (GTT) with $1 \mathrm{~g} / \mathrm{kg}$ glucose (Glucosteril, Fresenius, Bad Homburg, Germany) were performed in $4 \mathrm{~h}$ and $12 \mathrm{~h}$ fasted mice, respectively. Tail vein blood was used for measuring 


\section{Cellular Physiology Cell Physiol Biochem 2015;36:2357-2365 \begin{tabular}{ll|l}
\cline { 2 - 3 } and Bioch 10.1159/000430198 & $\begin{array}{l}\text { O 2015 S. Karger AG, Basel } \\
\text { www.karger.com/cpb }\end{array}$ \\
\hline
\end{tabular} \\ Wang/Zhang/Pan/Wang/Cao: MiR-499-5p Participates in Insulin Resistance}

the glucose concentration with a glucometer (Precision Xceed, Abbott, Wiesbaden, Germany) at the indicated time points.

\section{Cell culture}

Murine liver cell line NCTC1469 was cultured in Dulbecco's Modified Eagle's Medium (DMEM) supplemented with $10 \%(\mathrm{v} / \mathrm{v}$ ) Horse serum (Atlanta Biolabs), $100 \mathrm{units} / \mathrm{ml}$ penicillin, and $100 \mu \mathrm{g} / \mathrm{ml}$ streptomycin (Life Technologies, Inc.), at $37^{\circ} \mathrm{C}$ in a humidified atmosphere with $5 \% \mathrm{CO}_{2}$.

\section{RNA isolation and real-time PCR}

Total RNA was extracted from NCTC1469 cells with Trizol (Invitrogen) according to the manufacturer's instructions. The quality of the RNA samples was monitored by analysis of $\mathrm{OD}_{260} / \mathrm{OD}_{280}$.

To measure the level of miR-499-5p, a quantitative 2-step RT-PCR assay was performed using SYBR Green Supermix (Bio-Rad) in a BIO-RAD iCycler iQ real-time PCR detection system [13].

\section{Western blot analysis}

Cell lysates were separated by 10\% SDS-PAGE and were transferred to PVDF membranes (Millipore), blocked with $8 \%$ nonfat dry milk, and probed with antibodies at $4^{\circ} \mathrm{C}$ overnight. The blots were incubated with HRP-conjugated anti-IgG, followed by detection with ECL (Millipore). Antibodies against PTEN, phosphorylated AKT, GAPDH, GSK and phosphorylated GSK were purchased from Abcam. The appropriate HRP-conjugated anti-rabbit IgG secondary antibody (Abmart, all at 1:5000) was subsequently applied, and immunodetection was performed using the ECL Plus detection system (Millipore) according to the manufacturer's instructions. GAPDH was used as the internal control.

\section{Luciferase assay}

For luciferase assay, the 3' untranslated region (UTR) of PTEN including the binding sites for miR-499$5 p$ was amplified from NCTC1469 cells. PCR was performed with genome DNA isolated from NCTC1469 cells and the PCR product was then digested with XhoI (NEB). Then, the fragment was inserted into the Xhol-linearlized pGL3 (Promega) luciferase reporter vector. To ensure the ligation efficiency, both the PCR products and the XhoI-linearlized pGL3 vectors were treated with thermosensitive alkaline phosphatese (NEB) at $80^{\circ} \mathrm{C}$ for $20 \mathrm{~min}$. Details of PCR procedures are described as follows: a hot start step at $95^{\circ} \mathrm{C}$ for 10 min, followed by 40 cycles at $95^{\circ} \mathrm{C}$ for $15 \mathrm{~s}$ and $55^{\circ} \mathrm{C}$ for $45 \mathrm{~s}, 72{ }^{\circ} \mathrm{C}$ for $30 \mathrm{~s}$. Luciferase reporter assay was performed using Dual-Glo Luciferase assay system (Promega).

\section{Determination of glycogen content}

In the presence of $1 \mathrm{nM}$ insulin for $3 \mathrm{~h}$, the glycogen content was determined in the livers or NCTC1469 cells using a glycogen assay kit (Nanjing Jiancheng Bioengineering Institute, China) according to the manufacturer's instructions.

\section{Transfection of miRNA mimics or inhibitor}

MiR-499-5p mimics or inhibitor or negative control (NC) were purchased from Genepharm and transfected into NCTC1469 cells with a final concentration at $50 \mathrm{nM}$. The HiperFect transfection reagent was purchased from QIAGEN.

Inhibition of PTEN by RNA interference

PTEN-specific siRNA (siPTEN) and NC were purchased from Genepharma. $1 \times 10^{5}$ cells per well in a 6-well plate were transfected with $50 \mathrm{nM}$ siPTEN or NC for $48 \mathrm{~h}$ using HiperFect transfection reagent (QIAGEN) according to the structions.

\section{Statistics}

The data are represented as means \pm standard error (SE) of the indicated number of measurements. Differences were analyzed by Student's $t$-test with significance set at $P<0.05$. 
Table 1. The results of microRNA microarray analysis

\begin{tabular}{lllrcccc}
\hline Name & Fold change & \multicolumn{2}{l}{ ForeGround } & \multicolumn{2}{c}{ ForeGround-BackGround } & \multicolumn{2}{c}{ Normalized } \\
& (D/C) & C & D & C & D & C & D \\
\hline miR-499-5p & 0.11 & 168.5 & 77 & 104 & 11 & 0.41 & 0.04 \\
\hline
\end{tabular}

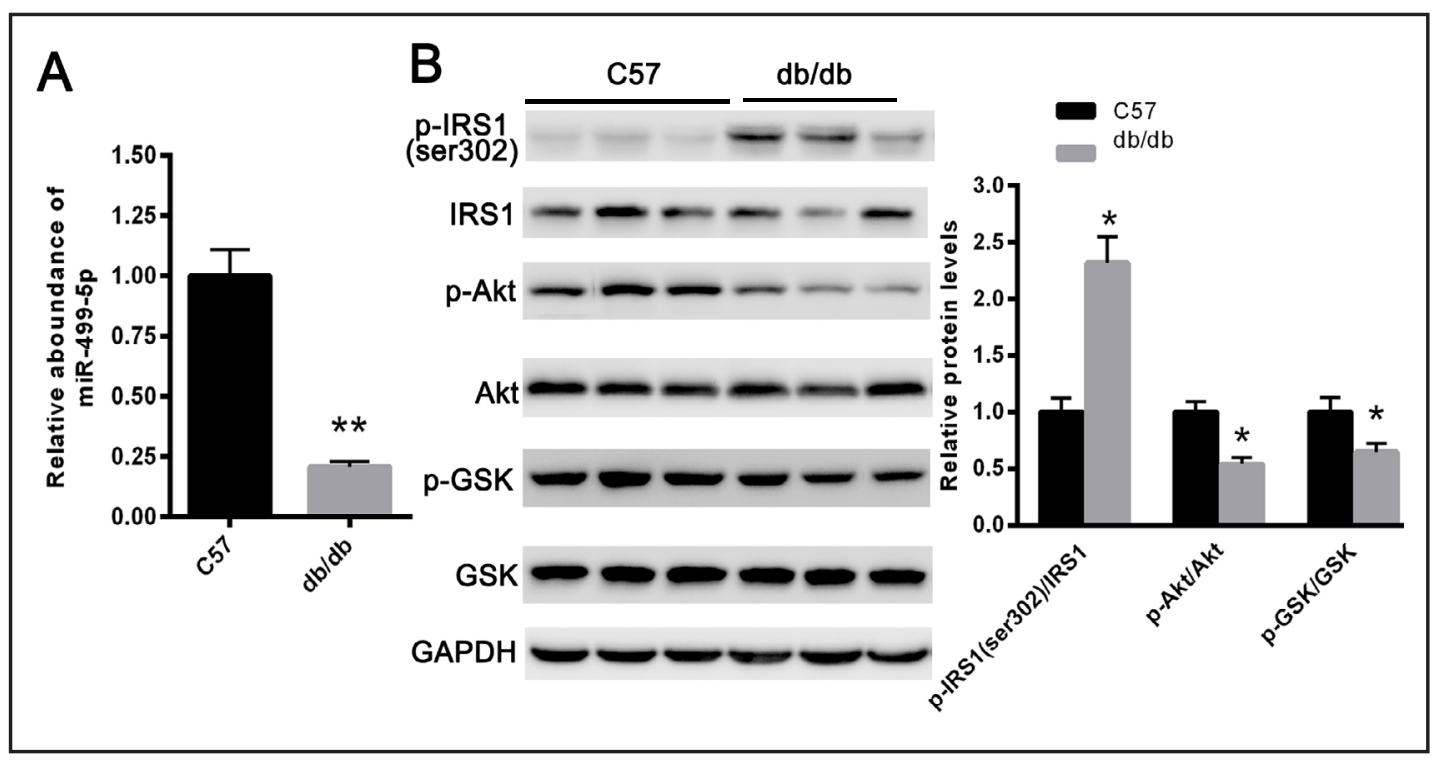

Fig. 1. miR-499-5p was decreased in the livers of db/db mice. (A) RT-qPCR was used to validate the level of miR-499-5p. (B) Western blot was applied to explore the activation of AKT/GSK. Data represent the means \pm S.E. (error bars), $\mathrm{n}=5$ independent experiments. ${ }^{*} \mathrm{p}<0.05$ versus control; ${ }^{* *} \mathrm{p}<0.01$ versus control.

\section{Results}

Decreased miR-499-5p levels in the livers of $d b / d b$ mice

The miRNA levels in the livers of $\mathrm{db} / \mathrm{db}$ mice were analyzed using a microarray assay. As shown in Table 1, the level of miR-499-5p was significantly decreased. The results were further validated using RT-qPCR (Fig. 1A). Moreover, we examined the signaling pathway of IRS1/PI3K/AKT in the livers of $\mathrm{db} / \mathrm{db}$ mice. We found the phosphorylation level of IRS1 at ser302 was obviously enhanced, while the activation of AKT and GSK was significantly decreased (Fig. 1B). These in vivo observations indicate that miR-499-5p may participate in hepatic insulin resistance.

miR-499-5p regulates the activation of the AKT/GSK signaling pathway in NCTC1469 cells

Next, the effect of miR-499-5p on hepatic insulin resistance was further explored in hepatocytes. The mimics of miR-499-5p were transfected into NCTC1469 cells for $48 \mathrm{~h}$. As shown in Fig. 2A, miR-499-5p was increased over fifty-fold compared with NC. Western blot revealed that overexpression of miR-499-5p in NCTC cells significantly activated the AKT/ GSK signaling pathway and increased the glycogen content (Fig. 2B and 2C). In comparison, transfection of a miR-499-5p inhibitor significantly decreased its level by 76\% in NCTC1469 cells (Fig. 2D). The levels of phosphorylated AKT/GSK were significantly inhibited, which was in concert with the reduced glycogen content (Fig. 2E and 2F). However, no changes of p-IRS1(ser302) were detected even when miR-499-5p was overexpressed or inhibited (Fig. 2B and 2E).Taken together, these results indicate that the reduced miR-499-5p level contributed to hepatic insulin resistance in the downstream signaling of PI3K/Akt pathway. 


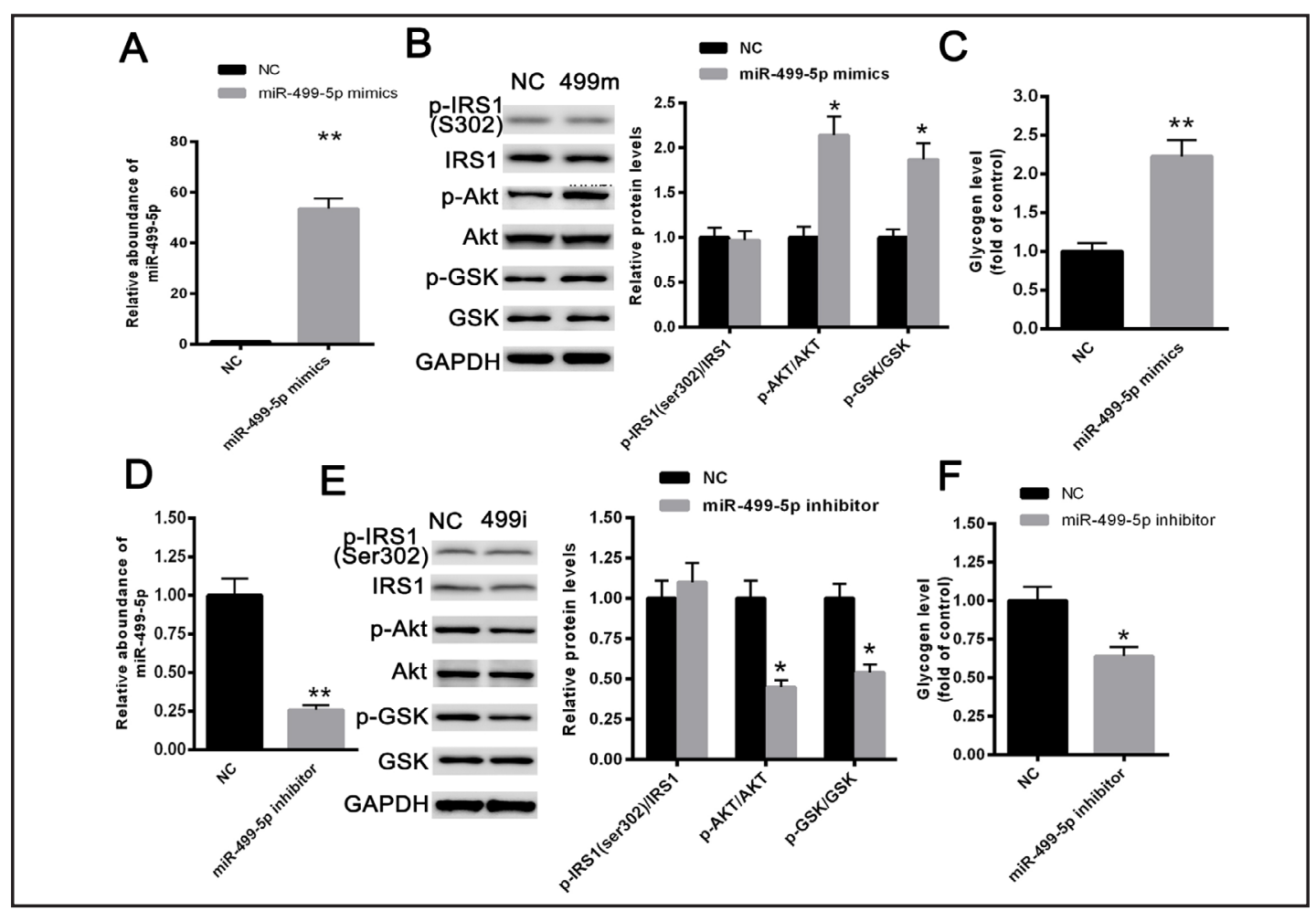

Fig. 2. miR-499-5p regulates the activation of AKT/GSK in NCTC1469 cells. (A) MiR-499-5p was increased over fifty-fold in NCTC1469 cells transfected with miR-499-5p mimics. (B) Overexpression of miR-499-5p prompted AKT/GSK activation and enhanced glycogen synthesis (C). (D) Transfection of a miR-499-5p inhibitor decreased its level by 76\% in NCTC1469 cells. Inhibition of miR-499-5p impaired the insulin signaling pathway (E) and reduced the glycogen content (F). Data represent the means \pm S.E. (error bars), $n=5$ independent experiments. ${ }^{*} \mathrm{p}<0.05$ versus control; ${ }^{* *} \mathrm{p}<0.01$ versus control.

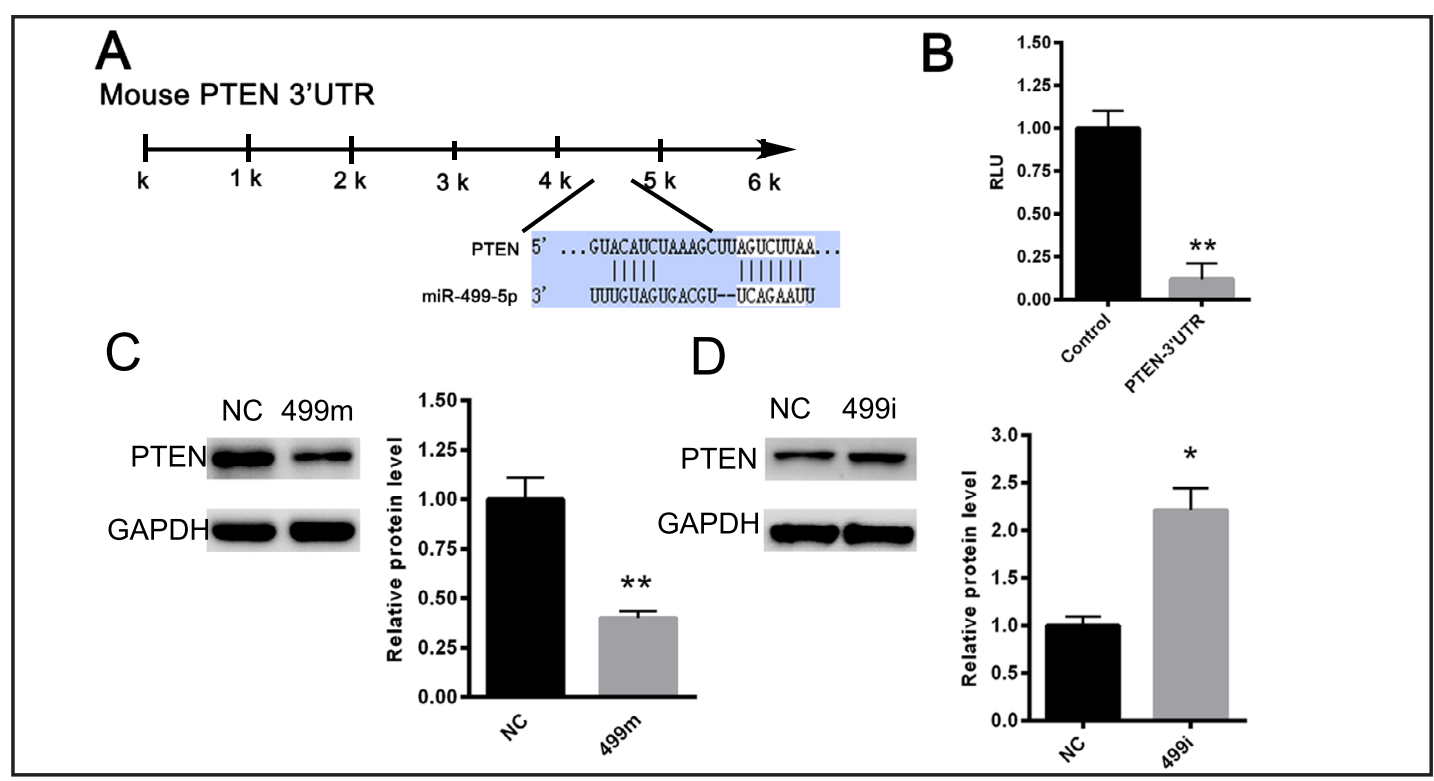

Fig. 3. PTEN was the target gene of miR-499-5p. (A) A bioinformatic assay revealed that PTEN was the target gene of miR-499-5p. (B) miR-499-5p significantly decreased PTEN-3'-UTR-pmiRGLO luciferase activity. Overexpression of miR-499-5p decreased the protein level of PTEN (C), and inhibition of miR-499-5p enhanced PTEN expression in NCTC1469 cells (D). Data represent the means \pm S.E. (error bars), $n=3$ independent experiments. ${ }^{*} \mathrm{p}<0.05$ versus control; ${ }^{* *} \mathrm{p}<0.01$ versus control. 


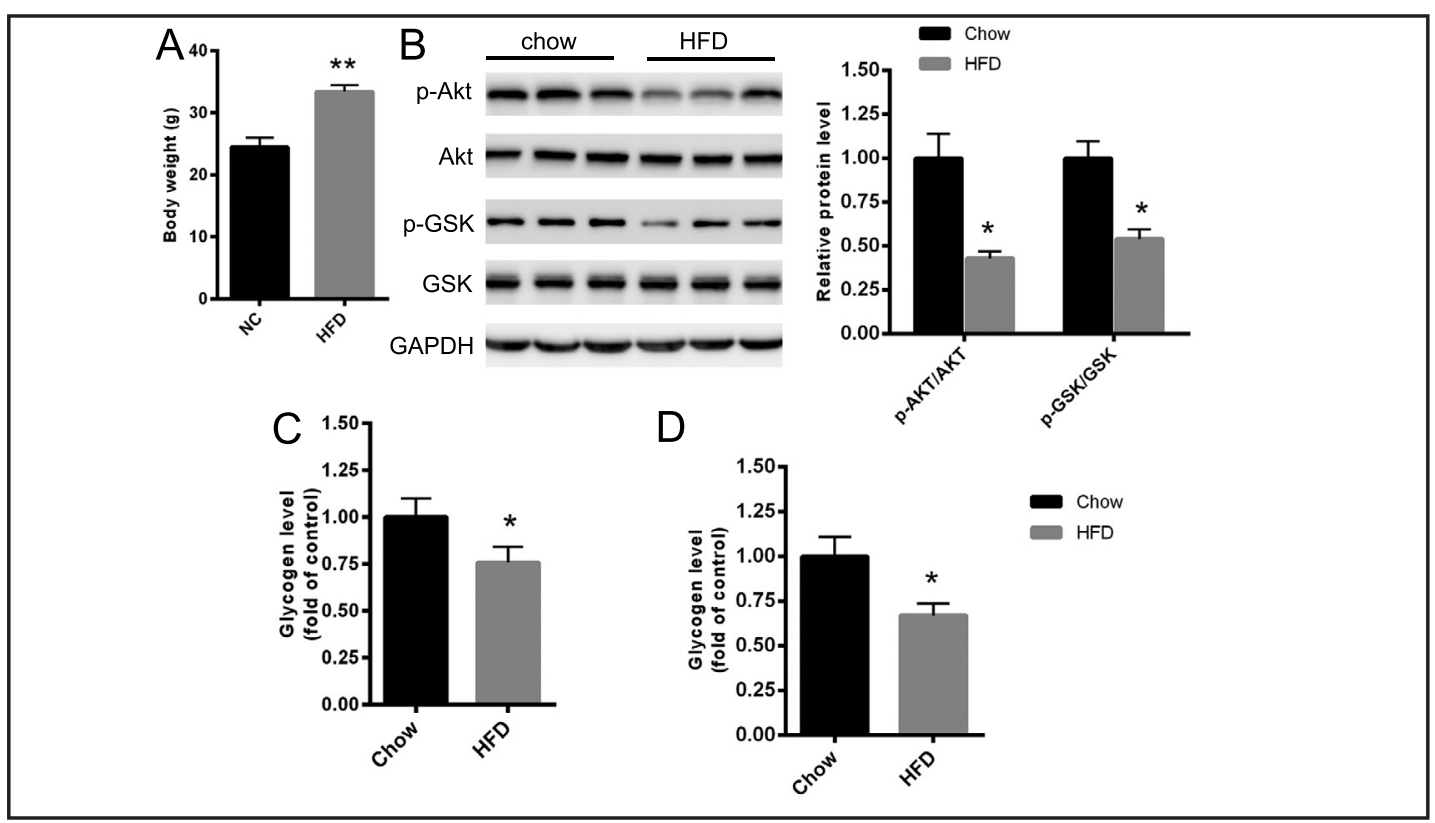

Fig. 4. miR-499-5p was reduced in the livers of HFD-fed mice. (A) Body weight was increased in the livers of HFD-fed mice compared with the control group. (B) The activation of AKT/GSK was significantly inhibited in HFD-fed mice. (C) The glycogen contents were decreased in the livers of HFD-fed mice. (D) The level of miR-499-5p was reduced by 34\% in the livers of HFD-fed mice. Data represent the means \pm S.E. (error bars), $\mathrm{n}=5$ independent experiments. ${ }^{*} \mathrm{p}<0.05$ versus control; ${ }^{* *} \mathrm{p}<0.01$ versus control.

\section{PTEN is the target gene of miR-499-5p}

To explore the specific mechanism of miR-499-5p in insulin resistance, bioinformatic analysis was applied to predict the target genes. As shown in the TargetScan analysis, PTEN was the target gene of miR-499-5p (Fig. 3A). Thus, the 3'-untranslated region (3'-UTR) of PTEN was cloned into a pmiRGLO plasmid. As shown in Fig. 3B, transfection of miR-499$5 p$ mimics significantly reduced the relative luciferase units of the PTEN-3'-UTR-pmiRGLO plasmid. More importantly, overexpression of miR-499-5p decreased the protein level of PTEN (Fig. 3C). Inhibition of miR-499-5p enhanced PTEN expression in NCTC1469 cells (Fig. 3D). These data suggest that PTEN is the target gene of miR-499-5p.

Reduced miR-499-5p level in the livers of HFD-fed mice

To further explore the role of miR-499-5p in insulin resistance, four-week-old male C57BL/6J mice were fed a standard laboratory diet or HFD for 10 weeks in a temperature and humidity-controlled environment. As shown in Fig. 4A, body weight increased compared with the control group. Moreover, the activation of AKT/GSK was significantly inhibited in the HFD-fed mice (Fig. 4B). Meanwhile, the glycogen contents were decreased in the livers of HFD-fed mice (Fig. 4C). We also explored the level of miR-499-5p in the livers of HFD-fed mice and found it was reduced by $34 \%$ (Fig. 4D).

Upregulation of miR-499-5p improved insulin resistance in HFD-fed mice

Recombinant adenoviruses expressing a miR-499-5p mimic (Ad-miR-499-5p-m) or a negative control adenovirus vector containing GFP (Ad-Con) were injected into the livers of mice through tail vein injection. As shown in Fig. 5A, the transfection efficiency of Ad-miR499-5p-m or Ad-Con was nearly the same in the livers of HFD-fed mice. Transfection of AdmiR-499-5p-m increased miR-499-5p by over 2.1-fold compared with the control group (Fig. 5B). Western blot analysis revealed that overexpression of miR-499-5p significantly reduced the PTEN level and activated AKT/GSK signaling in the livers of HFD-fed mice (Fig. 5C). Meanwhile, upregulation of miR-499-5p enhanced the glycogen contents in the livers of HFD- 


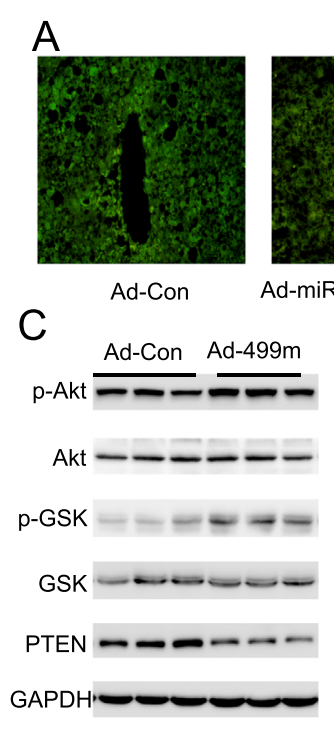

E
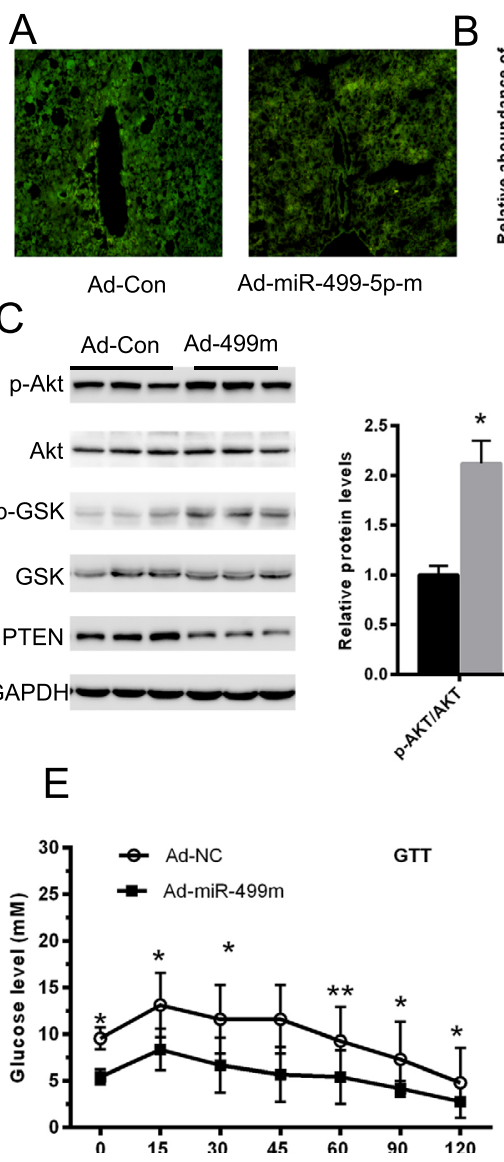

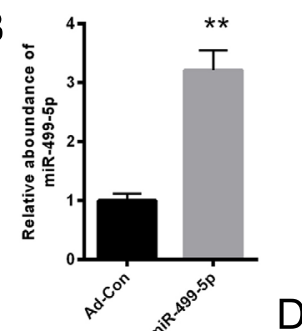

D

\section{D}

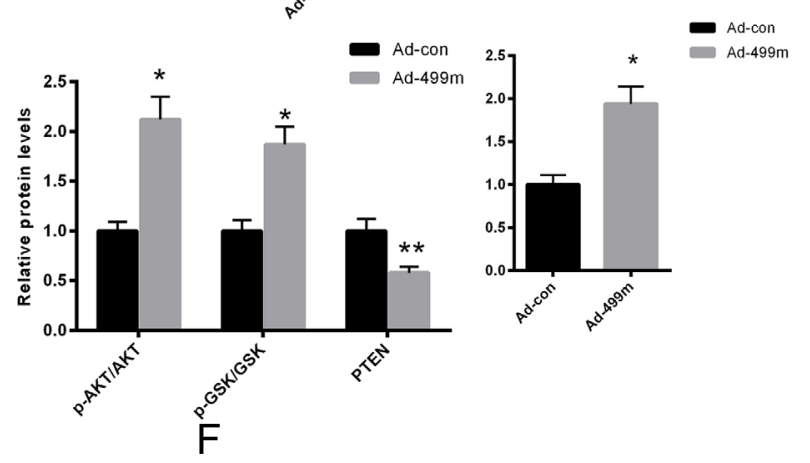

ITT

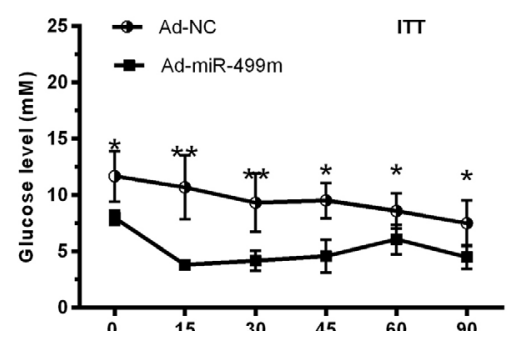

Fig. 5. Upregulation of miR-499-5p improved insulin resistance in HFD-fed mice. (A) Ad-miR-499-5p-m or Ad-Con were injected into the livers of mice through the tail vein, as indicated with GFP fluorescence. (B) Transfection of Ad-miR-499-5p-m increased miR-499-5p by over 2.1-fold compared with the control group. (C) Overexpression of miR-499-5p significantly reduced the PTEN level and activated AKT/GSK signaling in the livers of HFD-fed mice. (D) Upregulation of miR-499-5p enhanced glycogen contents in the livers of HFD-fed mice. GTT (E) and ITT (F) performance were measured in HFD-fed mice injected with Ad-miR-499$5 \mathrm{p}$-m or Ad-con. Data represent the means \pm S.E. (error bars), $\mathrm{n}=5$ independent experiments. ${ }^{*} \mathrm{p}<0.05$ versus control; ${ }^{* *} \mathrm{p}<0.01$ versus control.

fed mice (Fig. 5D). Furthermore, HFD-mice demonstrated better GTT and ITT performance when injected with Ad-miR-499-5p-m (Fig. 5E and 5F). These in vivo data further validated the protective role of miR-499-5p in insulin resistance.

\section{Discussion}

Increasing evidence has suggested that miRNAs play key roles in insulin resistance and are widely involved in the progression of type 2 diabetes $[14,15]$. In the present study, we found that miR-499-5p was reduced in the livers of $\mathrm{db} / \mathrm{db}$ mice and HFD-fed mice and was accompanied by impaired insulin signaling and glycogen synthesis. Further study revealed that overexpression of miR-499-5p improved insulin resistance in NCTC1469 cells, whereas inhibition of miR-499-5p reduced AKT/GSK activation. Our data demonstrate for the first time that reduced miR-499-5p levels in the liver contribute to hepatic insulin resistance.

Next, we explored the correlation between miR-499-5p and insulin resistance. According to TargetScan,PTEN was predicted as the targetgene of miR-499-5p. Previous studies indicated 


\section{Cellular Physiology Cell Physiol Biochem 2015;36:2357-2365 \begin{tabular}{l|l|l|}
\cline { 2 - 2 } DOI: 10.1159/000430198 & O 2015 S. Karger AG, Basel \\
\hline
\end{tabular}

that PTEN can dephosphorylate PI3K, thereby blocking the insulin signaling pathway [16]. Many studies have revealed the roles of microRNAs in regulating PTEN expression. For instance, miR-494 was found to target PTEN, contributing to EMT [17]. MiR-17-92 cluster enhances cholangiocarcinoma growth by regulating the downstream target, PTEN [18]. miR-499 was found to be expressed in the heart, and circulating miR-499 is suggested to be a sensitive biomarker for acute myocardial infarction [19]. It was also found to protect cardiomyocytes from $\mathrm{H}_{2} \mathrm{O}_{2}$-induced apoptosis by targeting Pdcd4 and Pacs2 [20]. However, no study has explored the role of miR-499 in hepatic insulin resistance. In the present study, we found a negative correlation between miR-499-5p and PTEN expression. To confirm the role of miR-499-5p in the repression of PTEN expression, miR-499-5p mimics, an inhibitor or a NC was transfected into NCTC1469 cells. Overexpression of miR-499-5p decreased PTEN expression, whereas inhibition of miR-499-5p enhanced PTEN levels. Furthermore, the luciferase reporter assay also demonstrated that miR-499-5p decreased the luciferase activity of PTEN-3'-UTR. The results showed that miR-499-5p negatively regulates AKT/GSK activation by targeting PTEN. It has been suggested that glucogenesis and lipogenesis are two major functions of livers [21]. In this study, we found that overexpression of miR-499$5 p$ in NCTC cells significantly increased the glycogen content, while inhibition of miR-499$5 p$ reduced the glycogen content. These data further suggested the role of miR-499-5p in hepatic insulin resistance.

Furthermore, we established an insulin resistance animal model using a HFD. We explored the level of miR-499-5p in the livers of HFD-fed mice and found it was enhanced. Second, we also validated the impaired insulin signaling pathway in the livers of HFD-fed mice compared with the control group. We performed a gain of function experiment for miR499-5p. Through tail vein injection of Ad-miR-499-5p-m or Ad-Con, hepatic miR-499-5p was increased more than 2-fold in the livers of HFD-fed mice compared with the control group. As a protein kinase, AKT significantly mediates insulin signaling for glucose metabolism [22]. Notably, hepatic overexpression of miR-499-5p significantly enhanced the glycogen contents and improved insulin signaling by reducing the PTEN expression level. Our data indicate that a reduced miR-499-5p level contributes to hepatic insulin resistance in HFDfed mice.

The current study demonstrates that miR-499-5p improves hepatic insulin sensitivity and prevents the progression of type 2 diabetes. Furthermore, miR-499-5p enhances the activation of the insulin signaling pathway mainly by targeting PTEN in liver cells and livers.

\section{Acknowledgments}

This study was supported by specialized research fund for the doctoral program of higher education (20123237120008).

\section{Disclosure Statement}

All authors have no conflicts of interest to declare.

\section{References}

1 McCarthy MI: Genomics, type 2 diabetes, and obesity. N Engl J Med 2010;363:2339-2350.

2 Barr EL, Zimmet PZ, Welborn TA, Jolley D, Magliano DJ, Dunstan DW, Cameron AJ, Dwyer T, Taylor HR, Tonkin AM, Wong TY, McNeil J, Shaw JE: Risk of cardiovascular and all-cause mortality in individuals with diabetes mellitus, impaired fasting glucose, and impaired glucose tolerance: The Australian Diabetes, Obesity, and Lifestyle Study (AusDiab). Circulation 2007;116:151-157. 


\section{Cellular Physiology Cell Physiol Biochem 2015;36:2357-2365 \begin{tabular}{l|l} 
and Biochemistry Published online: July 27, 2015 & $\begin{array}{l}\text { C) 2015 S. Karger AG, Basel } \\
\text { www.karger.com/cpb }\end{array}$ \\
\hline
\end{tabular} \\ Wang/Zhang/Pan/Wang/Cao: MiR-499-5p Participates in Insulin Resistance}

3 Dong Y, Gao G, Fan H, Li S, Li X, Liu W: Activation of the Liver X Receptor by Agonist T0901317 Improves Hepatic Insulin Resistance via Suppressing Reactive Oxygen Species and JNK Pathway. PLoS One. 2015;10:e0124778.

4 Peyrou M, Bourgoin L, Foti M: PTEN in non-alcoholic fatty liver disease/non-alcoholic steatohepatitis and cancer. Dig Dis 2010;28:236-246.

5 Vinciguerra M, Foti M: PTEN and SHIP2 phosphoinositide phosphatases as negative regulators of insulin signaling. Arch Physiol Biochem 2006;112:89-104.

6 Hodakoski C, Hopkins BD, Barrows D, Mense SM, Keniry M, Anderson KE, Kern PA, Hawkins PT, Stephens LR, Parsons R: Regulation of PTEN inhibition by the pleckstrin homology domain of P-REX2 during insulin signaling and glucose homeostasis. Proc Natl Acad Sci U S A 2014;111:155-160.

7 Ye EA, Steinle JJ: miR-15b/16 protects primary human retinal microvascular endothelial cells against hyperglycemia-induced increases in tumor necrosis factor alpha and suppressor of cytokine signaling 3. J Neuroinflammation 2015;12:44.

8 Wang R, Hong J, Cao Y, Shi J, Gu W, Ning G, Zhang Y, Wang W: Elevated circulating microRNA-122 is associated with obesity and insulin resistance in young adults. Eur J Endocrinol 2015;172:291-300.

9 de Guia RM, Rose AJ, Sommerfeld A, Seibert O, Strzoda D, Zota A, Feuchter Y, Krones-Herzig A, Sijmonsma T, Kirilov M, Sticht C, Gretz N, Dallinga-Thie G, Diederichs S, Klöting N, Blüher M, Berriel Diaz M, Herzig S: microRNA-379 couples glucocorticoid hormones to dysfunctional lipid homeostasis. EMBO J 2015;34:344360.

10 Feng YY, Xu XQ, Ji CB, Shi CM, Guo XR, Fu JF: Aberrant hepatic microRNA expression in nonalcoholic fatty liver disease. Cell Physiol Biochem 2014;34:1983-1997.

11 Wang G, Sun Y, He Y, Ji C, Hu B, Sun Y: MicroRNA-499-5p inhibits cell proliferation in hepatocellular carcinoma by target forkhead box P4 (FOXP4). Int J Clin Exp Pathol 2015;8:337-344.

12 Jacovetti C, Jimenez V, Ayuso E, Laybutt R, Peyot ML, Prentki M, Bosch F, Regazzi R: Contribution of intronic miR-499-5p and its Hosting Gene AATK to Compensatory $\beta$-cell Mass Expansion. Mol Endocrinol 2015;29:693-702.

13 Guay C, Regazzi R: Role of islet microRNAs in diabetes: which model for which question? Diabetologia 2015;58:456-463.

14 Chakraborty C, Doss CG, Bandyopadhyay S, Agoramoorthy G: Influence of miRNA in insulin signaling pathway and insulin resistance: micro-molecules with a major role in type-2 diabetes. Wiley Interdiscip Rev RNA 2014;5:697-712.

15 Kurtz CL, Peck BC, Fannin EE, Beysen C, Miao J, Landstreet SR, Ding S, Turaga V, Lund PK, Turner S, Biddinger SB, Vickers KC, Sethupathy P: MicroRNA-29 fine-tunes the expression of key FOXA2-activated lipid metabolism genes and is dysregulated in animal models of insulin resistance and diabetes. Diabetes 2014;63:3141-3148.

16 Peyrou M, Bourgoin L, Poher AL, Altirriba J, Maeder C, Caillon A, Fournier M, Montet X, Rohner-Jeanrenaud F, Foti M: Hepatic PTEN deficiency improves muscle insulin sensitivity and decreases adiposity in mice. J Hepatol 2015;62:421-429.

17 Yuan J, Benway CJ, Bagley J, Iacomini J: MicroRNA-494 Promotes Cyclosporine-Induced Nephrotoxicity and Epithelial to Mesenchymal Transition by Inhibiting PTEN. Am J Transplant 2015;15:1682-1691

18 Zhu H, Han C, Lu D, Wu T: miR-17-92 cluster promotes cholangiocarcinoma growth: evidence for PTEN as downstream target and IL-6/Stat3 as upstream activator. Am J Pathol 2014;184:2828-2839.

19 Zhang L, Chen X, Su T, Li H, Huang Q, Wu D, Yang C, Han Z: Circulating miR-499 are novel and sensitive biomarker of acute myocardial infarction. J Thorac Dis 2015;7:303-308.

20 Wang J, Jia Z, Zhang C, Sun M, Wang W, Chen P, Ma K, Zhang Y, Li X, Zhou C: miR-499 protects cardiomyocytes from H 20 2-induced apoptosis via its effects on Pdcd4 and Pacs2. RNA Biol 2014;11:339350.

21 Baranowski M, Zabielski P, Błachnio-Zabielska AU, Harasim E, Chabowski A, Górski J: Insulin-sensitizing effect of LXR agonist T0901317 in high-fat fed rats is associated with restored muscle GLUT4 expression and insulin-stimulated AS160 phosphorylation. Cell Physiol Biochem 2014;33:1047-1057.

22 Catherine P, Jean G: Contribution of de novo fatty acid synthesis to hepatic steatosis and insulin resistance: lessons from genetically engineered mice. J Clin Invest 2013;118:829-838. 